\title{
Combination therapy with interferon and ruxolitinib for polycythemia vera and myelofibrosis: are two drugs better than one?
}

\author{
Richard T. Silver
}

Richard T. Silver Myeloproliferative Neoplasms Center, Weill Cornell Medicine Division of Hematology-Oncology, New York, NY, USA

E-mail: RICHARD T. SILVER - rtsilve@med.cornell.edu

doi:10.3324/haematol.2020.256602

A pproximately 35 years ago, recombinant interferon alpha-2 (rIFN $\alpha$-2) was first reported to control myeloproliferation in essential thrombocythemia, ${ }^{1}$ polycythemia vera (PV), ${ }^{2}$ and the hyperproliferative phase of primary myelofibrosis (MF). ${ }^{3}$ In the ensuing years, these observations have been substantiated in thousands of patients with myeloproliferative neoplasms, indicating that rIFNo-2 is safe and effective for alleviating symptoms, diminishing organomegaly, reducing elevated platelet and white blood cell counts and, in PV, maintaining and controlling the hematocrit ${ }^{4}$ and decreasing the level of the JAK2 V617F allele burden. ${ }^{5}$ The effect of rIFN $\alpha$ on the molecular markers of essential thrombocythemia and MF have also been noted. ${ }^{6,7}$

The finding of a significant reduction of $J A K 2$ V617F level in a subset of PV patients treated with a long-acting form of interferon owing to its pegylation (PEG- rIFNa-2a) $)^{8}$ led to more enthusiastic use of this treatment. Subsequently, normalization of marrow morphology and reduction of fibrosis were demonstrated. ${ }^{9}$ Interferon monotherapy normalized elevated blood cell counts within a few months, but the major molecular responses and marrow changes required 3-5 years of treatment, especially in $M F^{10}$ Discontinuation of rIFN $\alpha$ in PV could be sustained for a number of years. ${ }^{3,8}$ Patients with primary or secondary $M F$ usually require continued treatment. ${ }^{10}$

As with all potent drugs used in treating hematologic cancers, rIFN $\alpha$ is associated with a significant number of side effects, but most commonly, constitutional symptoms such as fatigue, muscle aches, lethargy and, occasionally, fever. ${ }^{7}$

This is presumably due to cytokine effects. Discontinuation rates in rIFN $\alpha$ studies have ranged from $10-30 \%$ after $1-2$ years, ${ }^{4,7,8}$ depending on the dose and frequency of administration of the drug, the severity of associated side effects, and the belief and enthusiasm of the physicians and patients, respectively, regarding its value. Recently, it has been suggested that considering side effects to be all related to dosing, per se, is too simplistic. Danish investigators indicated that chronic inflammation induced by interferon renders patients intolerant or refractory to the treatment. ${ }^{11}$ Adding an anti-inflammatory drug with an anti-JAK2 V617F effect, such as ruxolitinib, seemed logical (Figure 1).
The study by Sørensen et al., reported in this issue of Haematologica comprised 50 patients with PV or with primary or secondary MF, all of whom were resistant or refractory to rIFN $\alpha-2 .{ }^{12}$ After the addition of ruxolitinib to the treatment regimen, of $32 \mathrm{PV}$ patients, ten (31\%) achieved complete or partial remission; of $18 \mathrm{MF}$ patients, eight (44\%) achieved complete or partial remission.

Combination treatment seemed to speed the time to remission, improve blood counts, reduce marrow cellularity and fibrosis and decrease the JAK2 allele burden, all with acceptable toxicity. The dropout rate at the end of 2 years was $6 \%$ for PV patients and $32 \%$ for MF patients.

These results are most interesting and encouraging, but require confirmation, because it was a single-arm study. Other limiting features, as the authors point out, include the small number of cases, the lack of a dose-finding phase, and the duration of only 2 years of treatment. Detailed molecular reporting of the MF patients in particular would have been of interest, since it is known that initial molecular profile affects response to interferon treatment in early $\mathrm{MF}^{10}$ Nevertheless, conceptually, the basis for the use of two potentially synergistic agents with different activities in untreated patients is logical. This then leads to the question of whether or not this combination should be used as initial therapy. The current tendency worldwide is to use hydroxyurea as firstline treatment when needed in PV and to adopt a watchand-wait attitude in treating primary MF. This approach seems illogical since it is not consistent with our concepts of cancer treatment in general, wherein treatment of early cancer yields results superior to those of treating metastatic disease. The reduction and/or elimination of symptoms of disease and avoidance of progression with agents that have a biological basis for use and that are tolerable seems far more rational. This requires testing in the immediate future.

\section{References}

1. Linkesch W, Gisslinger $H$, Ludwig $H$, Flener $R$, Sinzinger $H$. Therapy with interferon (recombinant IFN-alpha-2C) in myeloproliferative diseases with severe thrombocytoses.] Acta Med Austriaca. 1985;12(5):123-127. Article in German.

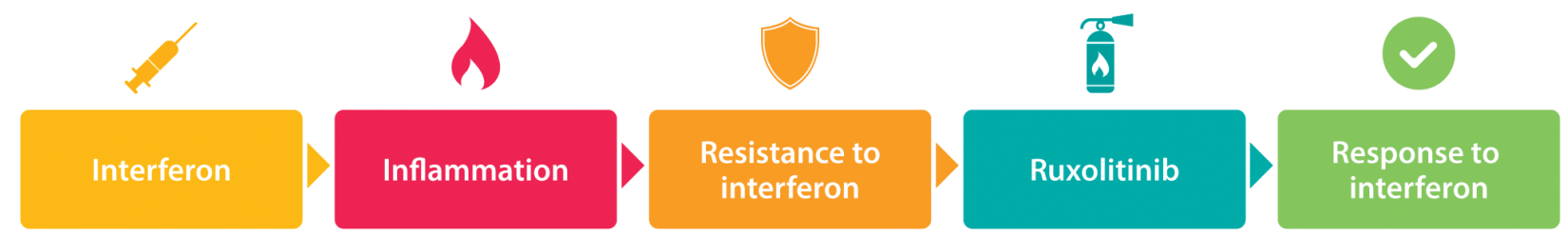

Figure 1. Overcoming inflammatory-resistance to interferon with ruxolitinib. 
2. Silver RT. Recombinant interferon-alpha for treatment of polycythaemia vera. Lancet. 1988;2(8607):403

3. Gilbert HS. Long term treatment of myeloproliferative disease with interferon-alpha-2b: feasibility and efficacy. Cancer. 1998;83(6): 1205-1213.

4. Silver RT. Long-term effects of the treatment of polycythemia vera with recombinant interferon-alpha. Cancer. 2006;107(3):451-458.

5. Jones AV, Silver RT, Waghorn K, et al. Minimal molecular response in polycythemia vera patients treated with imatinib or interferon alpha. Blood. 2006;107(8):3339-3341.

6. Barbui T, Tefferi A, Vannucchi AM, et al. Philadelphia chromosomenegative classical myeloproliferative neoplasms: revised management recommendations from European LeukemiaNet. Leukemia. 2018;32(5):1057-1069

7. Silver RT, Kiladjian JJ, Hasselbalch HC. Interferon and the treatment of polycythemia vera, essential thrombocythemia and myelofibrosis. Expert Rev Hem. 2013;6(1):49-58.
8. Kiladjian JJ, Cassinat B, Chevret S, et al. Pegylated interferon-alfa-2a induces complete hematologic and molecular responses with low toxicity in polycythemia vera. Blood. 2008;112(8):3065-3072.

9. Pizzi M, Silver RT, Barel AC, Orazi A. Recombinant interferon- $\alpha$ in myelofibrosis reduces bone marrow fibrosis, improves its morphology and is associated with clinical response. Mod. Pathol. 2015;28(10):1315-1323.

10. Silver RT, Barel AC, Lascu E, et al. The effect of initial molecular profile on response to recombinant interferon- $\alpha$ (rIFN $\alpha)$ treatment in early myelofibrosis. Cancer. 2017;123(14):2680-2687.

11. Mikkelsen SU, Kjaer L, Bjorn ME, et al. Safety and efficacy of combination therapy of interferon $\alpha-2$ and ruxolitinib in polycythemia vera and myelofibrosis. Cancer Med. 2018;7(8):3571-3581

12. Sørensen AL, Mikkelsen SU, Knudsen TA, et al. Ruxolitinib and interferon $\alpha 2$ combination therapy for patients with polycythemia vera or myelofibrosis: a phase II study. Haematologica. 2020;105(9): $2262-2272$

\section{Mysteries of partial dihydroorotate dehydrogenase inhibition and leukemia terminal differentiation}

\section{Yogen Saunthararajah}

Department of Translational Hematology and Oncology Research, Taussig Cancer Institute, Cleveland Clinic, Cleveland, OH, USA

E-mail: YOGEN SAUNTHARARAJAH - saunthy@ccf.org

doi:10.3324/haematol.2020.254482

I is reasonable to wonder why inhibiting dihydroorotate dehydrogenase $(\mathrm{DHODH})$, a protean and vital metabolic enzyme, would be expected to solve, not exacerbate, prevalent oncotherapy problems of toxicity and resistance. Yet, in addition to ASLAN003, described in this issue of Haematologica, ${ }^{1}$ at least four other $\mathrm{DHODH}$ inhibitors are being developed for oncotherapy. ${ }^{2} \mathrm{DHODH}$ is the sole mitochondrial enzyme in the pathway of de novo pyrimidine synthesis, which makes pyrimidine nucleobases from glutamine and aspartate. Pyrimidines are not just building blocks for DNA and RNA, but are also key cofactors for glycoprotein, glycolipid and phospholipid synthesis. Moreover, the reaction that DHODH executes, reduction of dihydroorotate to orotate, is coupled to mitochondrial electron transport, to manufacture ATP independently of glucose and the Krebs cycle. Not surprisingly, therefore, $\mathrm{DHODH}$ is vital - its knock-out is lethal. Surprisingly, however, treatment of malignant cells with clinically tolerable concentrations of DHODH inhibitors induces not the cytotoxicity (apoptosis) expected from most anti-metabolite oncotherapeutics but terminal differentiation.

Unbiased analyses illustrate this: of the thousands of genes most significantly up- and down-regulated by ASLAN003 treatment of acute myeloid leukemia (AML) cells, most are the same genes coordinately up- and downregulated during normal myeloid differentiation into granulocytes or monocytes (Figure 1A). Such ready recapitulation of normal lineage progression is rendered less astonishing upon recognition that malignant cells express very high levels of lineage differentiation-driving master transcription factors to begin with, e.g., SPI1, CEBPA, RUNX1 in AML cells., ${ }^{3,4}$ One function of these lineage master transcription factors is to activate lineage differentiation programs, but another is to cooperate with MYC for high-grade activation of proliferation - coupling of exponential proliferation and onward differentiation in this way is a feature of metazoan biology sometimes called 'transit amplification'. Oncogenic mutations decouple exponential proliferation from onward differentiation to create malignant self-replication. ${ }^{4}$ In short, partial DHODH inhibition reconnects circuitry already present to release malignant cells to complete lineage journeys already begun (Figure 1A).

This modality for leukemia/cancer cytoreduction is worthy of investment for three fundamental reasons. First, cell cycle exiting by terminal differentiation does not require the p53 apoptosis machinery that mediates cytoreduction by antimetabolite chemotherapeutics in general, and thus offers activity even in chemorefractory disease with p53system mutations. ${ }^{4}$ Second, DHODH inhibitor-mediated induction of terminal differentiation is not restricted to rare AML or genetic subtypes of cancer, although differences in pyrimidine metabolism between histologically diverse cancers may influence this activity (discussed below). Last but not least, non-cytotoxic differentiation-based oncotherapy can spare normal dividing cells essential for health/normal lifespan, offering a good therapeutic index. ${ }^{4}$ To efficiently realize these fundamentals in the clinic, however, an obvious question needs an answer: how exactly does partial inhibition of $\mathrm{DHODH}$, a protean metabolic enzyme, reconnect cancer cells to terminal lineage fates intended by their master transcription factor content?

Given the contributions of $\mathrm{DHODH}$ and pyrimidines to so many fundamental cellular functions, it is difficult to know where to begin to answer this question. Fortunately, work spanning decades has provided excellent clues. One important observation is that the small molecule cyclopentenyl cytosine (CPEC), which inhibits the last step in de novo pyrimidine synthesis, uridine triphosphate (UTP) amination into cytidine triphosphate (CTP) by CTP synthase 2 (CTPS2), also releases AML and solid tumor cancer cells to terminal lineage fates. ${ }^{5,6}$ Moreover, exogenous cytidine that restored CTP but not UTP pools, and exogenous uridine 